

\title{
REMOTE DETECTION SYSTEM
}

by

K. V. Nixon, S. W. France, C. Garcia, and R. D. Hastings

\begin{abstract}
A newiy designed remote detection system has been developed at LOS Alamos that allo:vs the collection of high-resolution gemma-ray spectra and neutron date from a remote location. The system consists of the remote unit and a command unit. The remote unit collects data in a potentielly hostile environment while the operator controls the unit by elther radio or wire link from a safe position. Both units are battery powered and are housed in metel cerrying cases.
\end{abstract}

\section{INTRODUCTION}

Operations that measure radiation fields may require long-term collection of gamma-ray and neutron data at multiple locationa. The need for personnel who operate the measurement equipment to be continuouly present over extended periods of time may subject them to higher-than-accepteble integrated doses of radiation. To reduce exposure to operating personnel in such situations, a remote detection system, sometimes called the Remote Pulse-Height Analyzer, was developed. The system consists of two units, approxirnately $43-$ by $46-$ by $30-\mathrm{cm}$ each, se shawn in Fig. 1. Shown in Fig. 2 is the remote unit that collects the neutron and gamme-ray data from the distant location, and
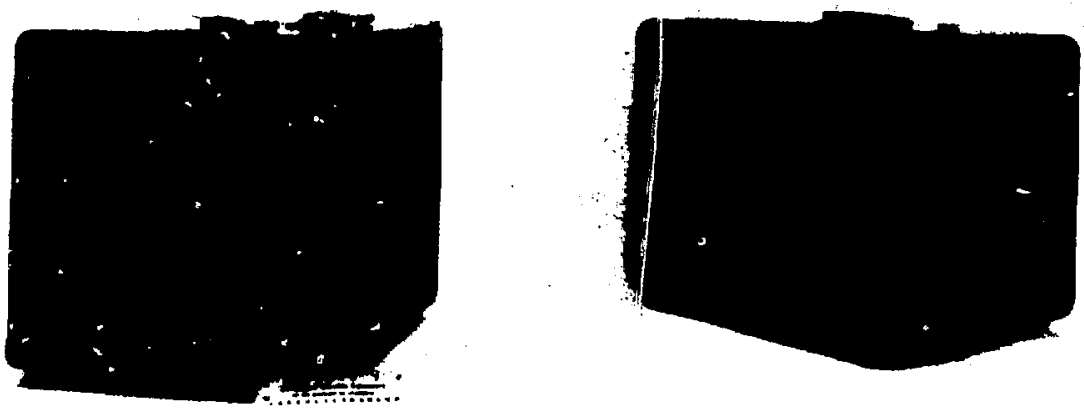

Fig. 1.

Remote datoction system. 
shown in Fig. 3 is the command unit that controls the operation from a safe location. Communication is via a HANDI-TALKI radio link or up to $300 \mathrm{~m}$ of thin control wire.

\section{GENERAL DESCRIPTION}

The remote unit, shown in Fig. 4, comprises a detector portion and a data collection portion. The reason for this partitioning will be explained later, but for now it should be noted that all of the electronics associated with detecting gamma rays and neutrons are packaged in one half of the unit and the remaining electronics in the other half.

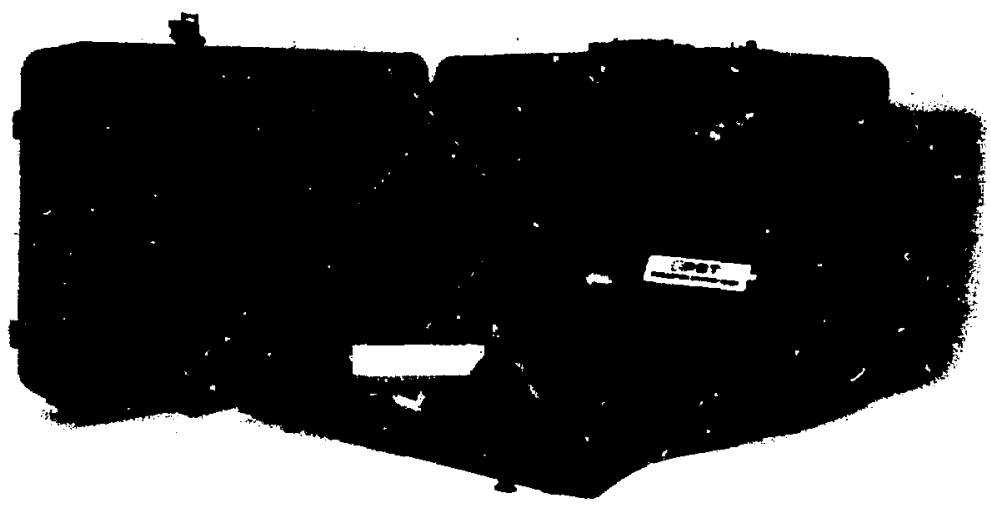

Fig. 2.

Remote unit.

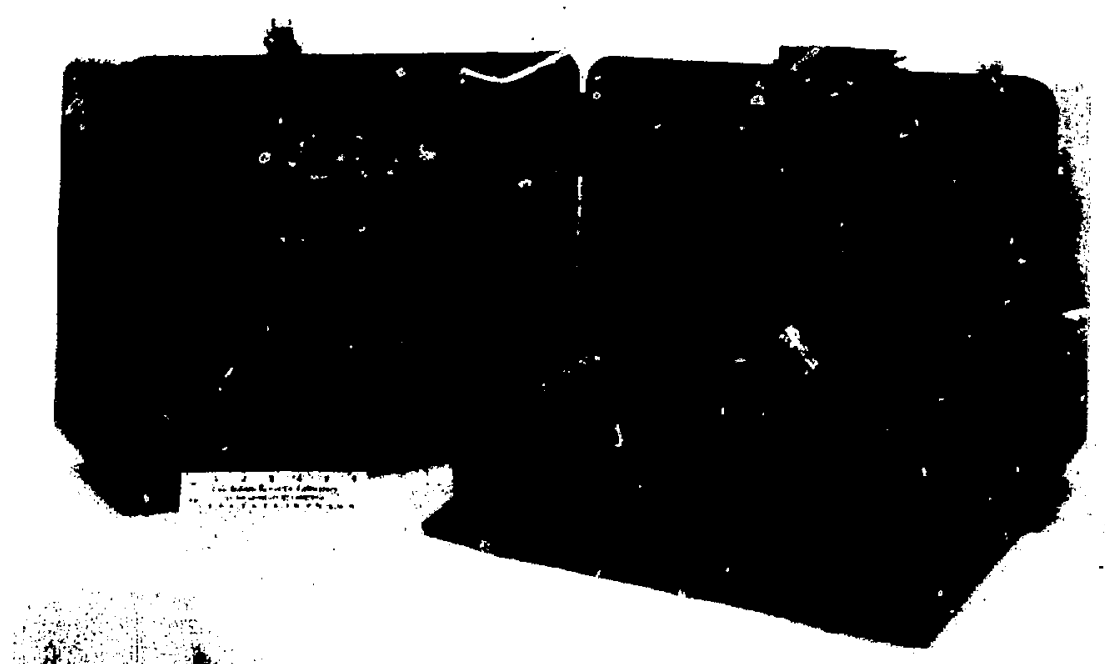

Fig. 3.

Command unit. 


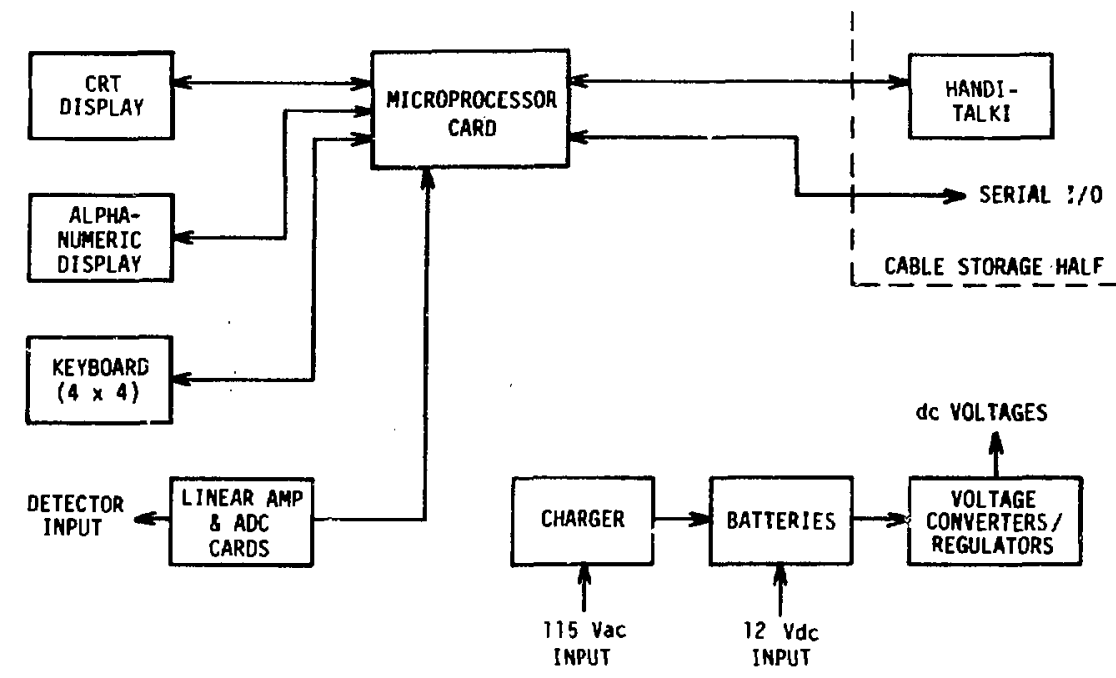

Fig. 4.

Remote block diagram.

The neutron detector consists of a ${ }^{3} \mathrm{He}(5-\mathrm{cm}$-diam by $30-\mathrm{cm})$ tube configured to reduce strong directional effects. A linear amplifier and discriminator are housed in a cylindrical extended socket configuration. The neutron discriminator output is a transistor-transistor logic (TTL) level that provides the input signal for the neutron scaler. The neutron scaler resides on the microprocessor card in the data collection portion of the remote unit. The scaler uses a crystal-derived clock to count seconds during an acquisition period. The neutron data, as well as the number of seconds, are transmitted to the command unit in channels $I$ and 0 , respectively.

The gamma detector is a high-purity germanium type with $22 \%$ efficiency. The high-voltage power supply is a Bertran PMT-SOAP (Option 3). The control circuits are designed so the high voltage will rise and fall at less than $500 \mathrm{~V} / \mathrm{sec}$ to protect the detector. The preamplifier and linear amplifier provide differential and integral linearity of better than $\pm 0.05 \%$ and amplify the signal to the $G-$ to $5-V$ input required by the analog-to-digitaI converter (ADC). The 4096-channel ADC collects the high-resolution spectral data that are stored in complementary metal-oxide-semiconductor (CMOS) random access memory. Long cables are provided (Fig. 5) for the all-attitude Dewar/detector/preamplifier unit to allow placement in more inaccessible locations.

Two manual operation buttons are provided on the remote unit to allow the operator to start and stop data acquisition without use of the command unit. A TEST button is also provided to ensure that the batteries have a good charge. An OFF/ON switch is provided and, although the system resets automatically when the power is turned on, a manual RESET button is provided. The mode switch selects either the HANDi-TALKI or the wire for serial communication.

The diagram of the command unit is shown in Fig. 6. The cathode-ray tube (CRT) display provides the operator with the standard pulse-height analyzer display of the spectral data. These data are obtained on command from the remote unit by way of the HANDI-TALKI or wire serial liniks. The keyboard is a 4 by 4 pushbutton type that serves as the control input. 


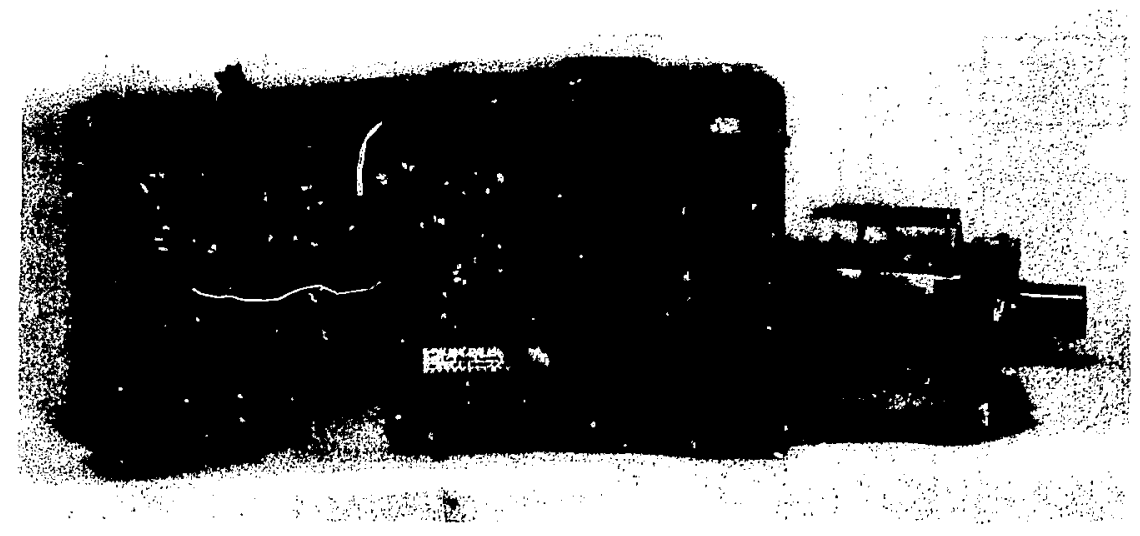

Fig. 5.

Remote alternative configuration.

A detailed description of the operating instructions follow, but for now it should be noted that the keyboard with the alpha-numeric ( $A / N)$ display provides the operator with the control input, the prompts, and the numerical readout. A 4096-channel ADC/linear amplifier is provided to allow the system to be reconfigured into a portable pulse-height analyzer. Figure 7 shows the system in this mode, which enables the gamma-ray signal to be input directly into the command unit through the ADC input. Such operation adds an extra degree of flexibility for the system with very little addition in hardware and only minor care with partitioning.

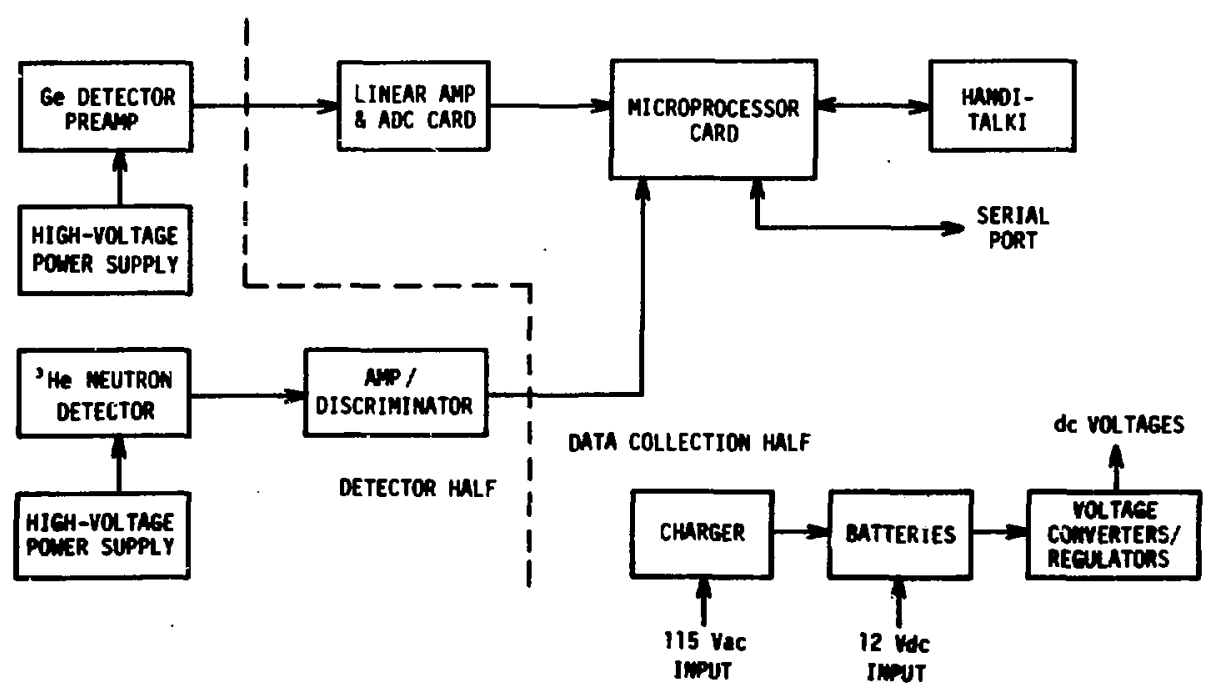

Fig. 6.

Command block diagram. 


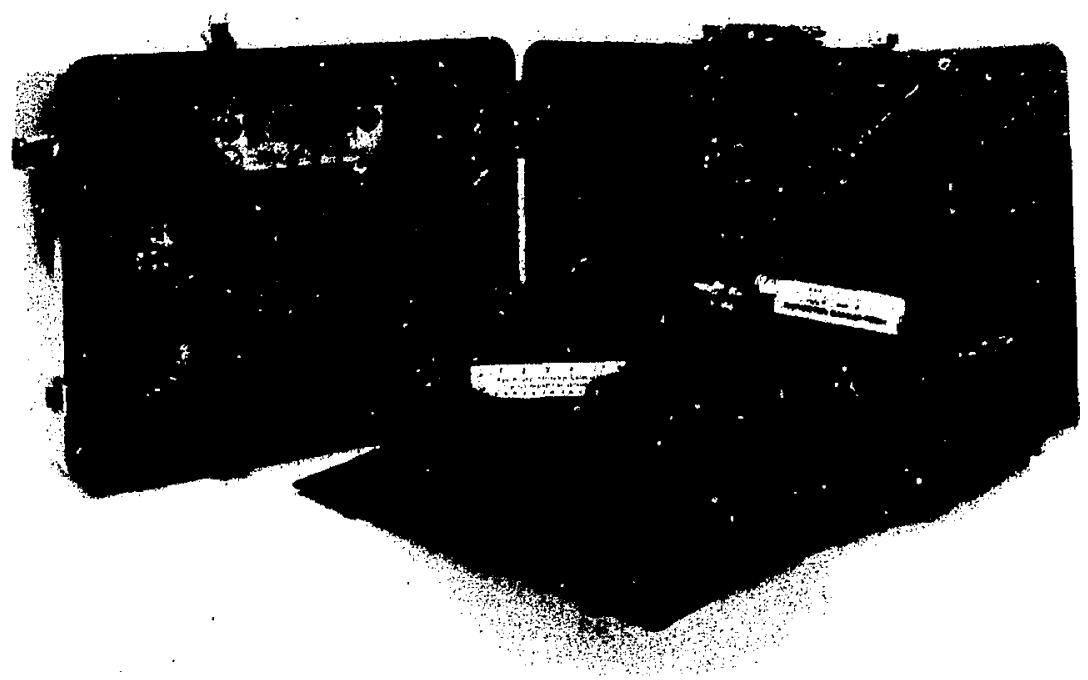

Fig. 7.

Portable pulse-height analyzer configuration.

Both units are provided with a very versatile power supply system. Two $4.5-\mathrm{amp} / \mathrm{hr}$ gel-cell batteries supply the $+12 \mathrm{Vdc}$ that is used to generate all of the other needed voltages. $A+12-V d c$ input is provided to allow operation of either system from an outside source such as the standard $+12-\mathrm{Vdc}$ car system. The internal $115-\mathrm{Vac}$ battery charger recharges the batteries in less than $12 \mathrm{hr}$. Space for additional batteries is available, however, the system will operate at least $4 \mathrm{hr}$ on the batteries provided.

\section{OPERATING INSTRUCTIONS}

The operation of the remote unit has been made very simple to allow operation by an individual with only minor training. This feature allows deployment by personnel unfamiliar with the equipment. An OFF/ON switch turns on the remote unit and automatically resets the microprocessor. A RESET button is provided to allow reset without cycling the power. A TEST button, when pushed, causes a light-emitting diode (LED) to light if the batteries are fully charged. A second LED lights when there is +12 Vdc provided to the batteries for charging. $A$ third LED indicates when +115 Vac is present. Two fuses for ac and dc short-circuit protection are provided. Manual ACQUIRE and STOP ACQUIRING buttons allow the operator to control operation locally if desired. In normal use, the operator need only place the unit at the desired location and turn it on. The power-on procedure can be done previously if time permits. When the system is in the wire-link mode, the wire must be connected to complete the placement task. All further control can be done from the command unit at a safe distance.

The control panel for the system is shown in Fig. 8. The controls in the upper right section are the standard scope controls and are associated with the CRT display. A detailed explanation can be found in the Tektronix Operator's Menual ${ }^{\prime}$ for the 221 oscilloscope, but operation of these controls is usually obvious to anyone familiar with scope operation. Table I summarizes the usua! setting of the controls during operation. 


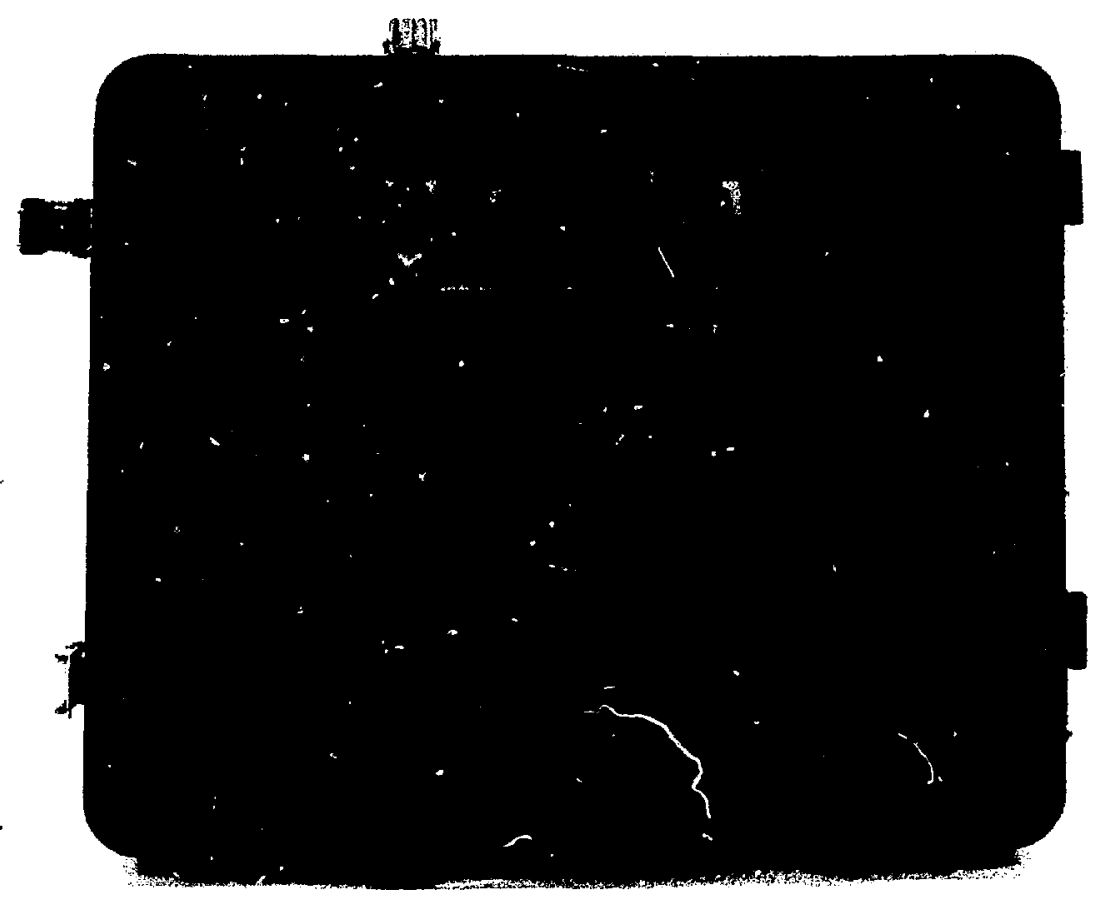

Fig. 8.

Control panel.

TABLE I

USUAL CONTROL SETTINGS DURING OPERATION

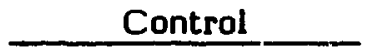

OFF/ON

Charınel 1 switch

Channel 2 switch

Channel 1 position

Channel 2 position

Channel 1 scale

Channel 2 scale

SEC/DIV

Trigger switch

Trigger potentiometer

Intensity

Horizontal magnification

Horizontal position
Setting

on

dc

dc

center display

(not applicable)

0.02 (V/division)

0.02 (V/division)

$X-Y$

(not applicable)

(not applicable)

---a

times 1

center display

Adjust until center channel appears slightly brighter than remaining channels. 
Located immediately below the oscilloscope are various controls and indicators associated with power for the unit. A TEST button with three LEDn indicates if the radio, logic, and oscilloscope batteries are sufficiently charged. Three additional LEDs indicate when the three battery chargers are operating. A three-position rotary switch selects power off, internal battery, or external +12 vdc. A connector is provided for external voltage hookup. A 115-Vac fuse protects the unit from internal overloads on the ac line. A 12-Vdc fuse is also provided for protection of the dc line. An LED indicates if the $115-V a c$ line is activated.

Immediately to the left of the power control section reside the controls for serial input and output to the modem. If the modem connector is in use, the six-position rotary switch selects the baud rate, and the serial signal is gated to that connector. When the modem switch is not in use, the serial port is controlled by the mode switch. When the radio switch is on, a 300 -baud rate is selected and the serial signal gated to the radio. When the radio switch is off, a 1200-baud rate is set, and the signal is gated to the remote wire connector. Thus, the serial port can be used for three separate outputs. A RESET button allows the microprocessor to be reinitialized without cycling the power off.

The keyboard provid ss most of the control for the entire system. Figure 9 and Table II summarize the keyboard operation. Keys 0-7 control the acquisition and transmission of data whereas keys 8-15 are used to control the display of the data. Keys $0,1,2,4$, and 6 operate the same in both the remote and command unit. If used by themselves, they control data locally in the portable pulse-height mode. If, however, the remote key (7) is pressed first, the commands are transmitted over the serial port to the remote unit for data control of that unit. Key 3 is the memory test key and, when used with the remote key, causes the remote unit to write the address of each channel into its memory location and then read each to verify accuracy. If all locations are correct, the complement of the address is written into each location and subsequently verified. If this :sst is also passed, a signal is sent to the command unit, which then responds MEMO OK with the A./N display.

\begin{tabular}{|c|c|c|c|}
\hline$A C Q$ & $\begin{array}{l}1 \\
\text { STP } \\
\text { ACQ }\end{array}$ & $\begin{array}{l}{ }^{2} \\
\text { CLR } \\
\text { MEM }\end{array}$ & $\begin{array}{l}{ }_{\text {MEM }}{ }^{3} \\
\text { TST }\end{array}$ \\
\hline${ }^{4}$ & $\begin{array}{r}5 \\
\text { (spare) }\end{array}$ & $\begin{array}{l}\text { ECO } \\
\text { TST }\end{array}$ & $\begin{array}{r}7 \\
\text { REMOTE }\end{array}$ \\
\hline$\underset{\operatorname{Exp}}{8}$ & $\begin{array}{l}9 \\
{ }^{\text {CNT }} \\
-x-\end{array}$ & $\begin{array}{l}\text { MRKR } \\
\text { LEFT } \\
\end{array}$ & $\begin{array}{l}\text { MRKR } \\
\text { RGHT } \\
\square\end{array}$ \\
\hline${ }_{\#}^{\text {CHNL }}{ }^{12}$ & $\stackrel{\#}{\text { CNTS }}^{13}$ & $\begin{array}{r}14 \\
\text { (spare) }\end{array}$ & $\begin{array}{r}15 \\
\text { (spare) }\end{array}$ \\
\hline
\end{tabular}

Fig. 9.

Keyboard layout. 
TABLE II

COMMAND SUMMARY

\begin{tabular}{cll} 
Key Number & \multicolumn{1}{c}{ Command } & \multicolumn{1}{c}{ Comments } \\
\cline { 2 - 3 } & Acquire data & Starts acquisition of data \\
1 & Stop acquisition & Stops system from acquiring data \\
2 & Clear memory & Clears data memory \\
3 & Memory test & For remote unit only \\
4 & Output & Outputs data to serial port \\
5 & Spare & - \\
6 & Echo test & - \\
7 & Remoie & Sends next keystroke out serial port \\
8 & Expand & Expands CRT horizontal display \\
9 & Contract & Contracts CRT horizontal display \\
$10^{a}$ & Marker left & Moves marker to the left (hence, CRT \\
$11^{a}$ & Marker right & display moves to right) \\
$12^{a}$ & Display channel & Moves marker to the right (hence, CRT \\
$13^{a}$ & display moves to left) \\
14 & Display count & Display marker channel number on \\
15 & Spare & A/N display \\
& Spare & Display count at marker on A/N
\end{tabular}

akeys 12 and 13 control the $A / N$ display. Key 12 causes the channel number of the marker to be shown and key 13 displays the number of counts in the marker channel. If the marker motion keys are pressed, the new channei numbers are displayed automatically。 The $A / N$ display has numerous other responses or prompts, which are summarized in Table III.

TABLE III

ALPHA-NUMERIC SUMMARY

Alpha-Numeric Display

ACQ DATA

STOP ACQ

READY

TEST OK

$\mathrm{CT}=$

CHNL =
Description

After data acquisition has started

After data acquisition has stopped

After power on or reset

After unit successfully passes ECHO, REMOTE ECHO, or REMOTE MEMORY TEST

After display count has been pressed

After display channel number has been pressed 
The output key (4), when pressed, transmits spectral data through the modem port in a format compatible with the Tracor Northern 1710 Pulse-Height Analyzer. Thus, the data can be stored on tape to be read into a 1710 analyzer later or read into a 1710 analyzer directly over a modem link. If the rernote key (7) is pressed prios to the output key (4), the output command is transmitted to the remote unit, which then transmits all of its spectral data over the serial link. This transmission is in simpie binary format that decreases the transmit time by a factor of 4 .

The echo test key (6) provides testing of the serial port. When used by itself, a word is sent out by the universal asynchronous receiver/transmitter (UART) and modem, which echoes it back to test internal circuitry. When the remote key is used prior to the echo command, the test word is transmitted to the remote unit, which echoes it back to allow testing of the serial link between units. The remote commarid, when used with keys 7 through 15, will result in no action.

Keys 8 through 13 control the display of spectral data. Key 8 causes the horizontal scale of the CRT display to expand to the next largest scale. The three scales available are 4096, 1029, and 128 channels (full scale). The CONTRACT key (?) causes the horizontal scale to change to the next smaller scale. The vertical scale is automatically changed to enable the largest channel within the display to be within the view. The MARKER LEFT key (10) causes the marker (center channel in the display) to move to the left with respect to the spectrum. The speed of travel is faster for the larger horizontal scales to enable quick coverage of the entire spectrum. The MARKER RIGHT key (II) operates similarly, except that the spectrum moves in the opposite directiori.

\section{SUMMARY}

The need frjr a versatile data collection system for neutron pulses and gamrna-ray spectra has been recognized and a viable solution found. The remote detection system has been tested and found to be a valuable tool in the field. High-resolution gamma-ray and neutron data have been acquired remotely and have enabled diagnostic operation to continue while keeping personnel at a safe distance.

\section{ACKNOWLEDGMENT}

We would like to thank Carl N. Henry for his help early in the sesign and develcpment.

\section{REFERENCE}

1. Tektronix 221 Oscilloscope Operator's Instruction Manual, Tektronix 070-1572-00. 